\title{
A Fuzzy Particle Swarm Approach to Multiobjective Quadratic Assignment Problems
}

\author{
Mingyan Zhao ${ }^{1}$, Ajith Abraham ${ }^{2}$, Crina Grosan ${ }^{3}$ and Hongbo Liu ${ }^{1,4}$ \\ ${ }^{1}$ School of Software, Dalian University of Technology, 116620 Dalian, China. \\ ${ }^{2}$ Centre for Quantifiable Quality of Service in Communication Systems, \\ Norwegian University of Science and Technology, Trondheim, Norway. \\ ajith.abraham@ieee.org \\ ${ }^{3}$ Department of Computer Science, Faculty of Mathematics and Computer Science \\ Babes-Bolyai University, Cluj-Napoca, Romania \\ ${ }^{4}$ School of Computer Science and Engineering, Dalian Maritime University, 116026 Dalian, China. \\ lhb@dlut.edu.cn
}

\begin{abstract}
The multiobjective Quadratic Assignment Problem (mQAP) is considered as one of the hardest optimization problems but with many real-world applications. Since it may not be possible to simply weight the importance of each flow for the $m Q A P$, it is best to use Pareto optimization to obtain the Pareto front or an approximation of it. Although Particle Swarm Optimization (PSO) algorithm has exhibited good performance across a wide range of application problems, research on $m Q A P$ has not much been investigated. This paper introduces a fuzzy particle swarm algorithm to handle the Multiobjective Quadratic Assignment Problem (mQAP). In the fuzzy scheme, the representations of the position and velocity of the particles in the conventional PSO is extended from the real vectors to fuzzy matrices. A new mapping is introduced between the particles in the swarm and the problem space in an efficient way. We evaluated the performance of the proposed approach. Empirical results illustrate that the approach can be applied for solving $m Q A P$ 's very effectively.
\end{abstract}

\section{Introduction}

The scalar quadratic assignment problem (QAP) was introduced in 1957 by Koopmans and Beckmann [1]. In 2002, Knowles and Corne extended the QAP to be multiobjective and it became the multiobjective quadratic assign- ment problem [2]. Since then, it has several good practical applications in diverse areas, such as some layout problems, network design problems, and communication problems $[3,4,5]$. The QAP is a NP-hard optimization problem and instances of size larger than 20 are considered intractable. A literature review reveals the use of stochastic local search, genetic algorithms (GA) and ant colony optimization to find some good solutions within a reasonable amount of time.

Particle swarm optimization (PSO) incorporates swarming behaviors observed in flocks of birds, schools of fish, or swarms of bees, and even human social behavior, from which the swarm intelligence paradigm has emerged [6, 8]. The main strength of PSO is its fast convergence, which compares favorably with many global optimization algorithms [9]. However, research for solving the mQAPs using PSO approach has not much been investigated. In this paper, we make an attempt to solve the problem using a discrete particle swarm optimization approach based on a fuzzy scheme and a kind of matrix mapping, which help us to deal with the constraints in the problems and provide more diverse solutions for the problems.

The rest of the paper is organized as follows. We formulate the quadratic assignment problem and multiobjective quadratic assignment problem in Section 2. The proposed fuzzy particle swarm approach for mQAP is presented in Section 3. Experiment, results and discussions are given in Section 4 followed by some conclusions in the last Section. 


\section{QAP and mQAP}

The QAP can be described as the problem of assigning a set of facilities to a set of locations with given distances between the locations and given flows between the facilities. The goal then is to place the facilities on locations in such a way that the sum of the product between flows and distances is minimal [10]. More formally, given $n$ facilities $\left\{F_{1}, F_{2}, \cdots, F_{n}\right\}$ and $n$ locations $\left\{L_{1}, L_{2}, \cdots, L_{n}\right\}$, two $n \times n$ matrices $F M=\left[f_{i j}\right]$ and $D M=\left[d_{r s}\right]$, where $f_{i j}$ is the flow between facilities $F_{i}$ and $F_{j}$ and $d_{r s}$ is the distance between locations $L_{r}$ and $L_{s}$, the QAP can be stated as follows:

$$
\min _{\pi \in P(n)} C(\pi)=\sum_{i=1}^{n} \sum_{j=1}^{n} f_{i j} d_{\pi_{i} \pi_{j}}
$$

where $P(n)$ is the set of all permutations (corresponding to the assignment solutions) of the set of integers $\{1,2, \cdots, n\}$, and $\pi_{i}$ gives the location of facility $F_{i}$ in the current solution $\pi \in P(n)$. Here $f_{i j} d_{\pi_{i} \pi_{j}}$ describes the cost contribution of simultaneously assigning facility $F_{i}$ to location $\pi_{i}$ and facility $F_{j}$ to location $\pi_{j}$. It is to be noted that the number of facilities $(n)$ is assumed to be the same as the number of locations. In other words, one facility could be assigned to only one location, and one location could be assigned to only one facility in a feasible assignment solution.

The term quadratic stems from the formulation of the QAP as an integer optimization problem with a quadratic objective function [10]. Let $b_{i j}$ be a binary variable which takes value 1 if facility $F_{i}$ is assigned to location $L_{j}$ and 0 otherwise. Then the problem can be re-formulated as:

$$
\min \sum_{i=1}^{n} \sum_{j=1}^{n} \sum_{r=1}^{n} \sum_{s=1}^{n} f_{i j} d_{r s} b_{i r} b_{j s}
$$

s.t.

$$
\begin{gathered}
b_{i j} \in\{0,1\}, \quad i=1,2, \cdots, n, \quad j=1,2, \cdots, n ; \\
\sum_{i=1}^{n} b_{i j}=1, \quad j=1,2, \cdots, n ; \\
\sum_{j=1}^{n} b_{i j}=1, \quad i=1,2, \cdots, n .
\end{gathered}
$$

Knowles and Corne [2] presented another QAP variation (mQAP) considering several flow and distance matrices. For the allocation of facilities in hospitals, it is desired to minimize the products of the flows by the distances between doctors and patients, and between nurses and medical equipment simultaneously. Each of the matrices describing these flows is very different and it may not be possible to simply weight the importance of each flow. It can be modeled using QAP but as a multiobjective problem. Formally, it can be defined mathematically as:

$$
\min _{\pi \in P(n)} \vec{C}(\pi)=\left\{C^{1}(\pi), C^{2}(\pi), \cdots, C^{m}(\pi)\right\}
$$

where

$$
C^{k}(\pi)=\sum_{i=1}^{n} \sum_{j=1}^{n} f_{i j}^{k} d_{\pi_{i} \pi_{j}}, \quad 1 \leq k \leq m .
$$

In this last constraint, $f_{i j}^{k}$ denotes the $k$ th flow between $i$ and $j$-facilities, others are similar to the above definition in the QAP.

Since it may not be possible to simply weigh the importance of each flow for the mQAP, the solution usually must be non-dominated and it is impossible to improve one component of the solution without worsening the value of at least one other component of the solution [2]. We must deal with generating the set of non-dominated solutions for the problems having more than one objective. So the Pareto optimization is used to obtain the Pareto front or an approximation of it.

\section{A Fuzzy Particle Swarm Approach for mQAP}

The classical particle swarm model consists of a swarm of particles, which are initialized with a population of random candidate solutions. They move iteratively through the $d$-dimension problem space to search new solutions, where the fitness $f$ can be calculated as the certain quality measure. Each particle has a position represented by a positionvector $\vec{x}_{i}$ ( $i$ is the index of the particle), and a velocity represented by a velocity-vector $\vec{v}_{i}$. Each particle remembers its own best position so far in a vector $\vec{x}_{i}^{\#}$, and its $j$-th dimensional value is $x_{i j}^{\#}$. The best position-vector among the swarm so far is then stored in a vector $\vec{x}^{*}$, and its $j$-th dimensional value is $x_{j}^{*}$. During the iteration time $t$, the update of the velocity from the previous velocity to the new velocity is determined by Eq.(8). The new position is then determined by the sum of the previous position and the new velocity by Eq.(9).

$$
\begin{gathered}
v_{i j}(t+1)=w v_{i j}(t)+c_{1} r_{1}\left(x_{i j}^{\#}(t)-x_{i j}(t)\right) \\
+c_{2} r_{2}\left(x_{j}^{*}(t)-x_{i j}(t)\right) \\
x_{i j}(t+1)=x_{i j}(t)+v_{i j}(t+1)
\end{gathered}
$$

Where $r_{1}$ and $r_{2}$ are the random numbers in the interval $[0,1] . c_{1}$ is a positive constant, called as coefficient of the self-recognition component, $c_{2}$ is a positive constant, called as coefficient of the social component. The variable $w$ is 
called as the inertia factor, which value is typically setup to vary linearly from 1 to near 0 during the iterated processing. From Eq.(8), a particle decides where to move next, considering its current state, its own experience, which is the memory of its best past position, and the experience of its most successful particle in the swarm.

For applying the particle swarm algorithm successfully for a problem, one of the key issues is how to map the problem solution to the particle space, which directly affects its feasibility and performance. In a "crisp" particle swarm model for the assignment problem, it would trend to assign many facilities to the same location or assign many locations to the same facility. This kind of assignment would be unfeasible. In this section, a fuzzy matrix is introduced to represent the quadratic assignment problem [11]. Then a PSO approach to the problem space mapping is depicted for the multiobjective quadratic assignment problem. Suppose $F=\left\{F_{1}, F_{2}, \cdots, F_{n}\right\}, L=\left\{L_{1}, L_{2}, \cdots, L_{n}\right\}$, then the fuzzy assignment relation from $F$ to $L$ is expressed as follows:

$$
A=\left[\begin{array}{cccc}
a_{11} & a_{12} & \cdots & a_{1 n} \\
a_{21} & a_{22} & \cdots & a_{2 n} \\
\vdots & \vdots & \ddots & \vdots \\
a_{n 1} & a_{n 2} & \cdots & a_{n n}
\end{array}\right]
$$

Here $a_{i j}$ represents the degree of membership of the $j$-th element $F_{j}$ in domain $F$ and the $i$-th element $L_{i}$ in domain $L$ to relation $A$. In the fuzzy relation matrix $A$ between $F$ and $L$, the elements are subject to the following constraints:

$$
a_{i j}=\mu_{R}\left(F_{j}, L_{i}\right), \quad i=1,2, \cdots, n, \quad j=1,2, \cdots, n .
$$

$\mu_{R}$ is the membership function, the value of $a_{i j}$ means the degree of membership that the facility $F_{j}$ would be assigned to the location $L_{i}$ in the feasible assignment solution. In the quadratic assignment problem, the elements of the solution must satisfy the following conditions:

$$
\begin{gathered}
a_{i j} \in[0,1], \quad i=1,2, \cdots, n, \quad j=1,2, \cdots, n \\
\sum_{i=1}^{n} a_{i j}=1, \quad j=1,2, \cdots, n \\
\sum_{j=1}^{n} a_{i j}=1, \quad i=1,2, \cdots, n .
\end{gathered}
$$

According to fuzzy matrix representation of the quadratic assignment problem, the position $X$ and velocity $V$ in the particle swarm are re-defined as follows:

$$
X=\left[\begin{array}{cccc}
x_{11} & x_{12} & \cdots & x_{1 n} \\
x_{21} & x_{22} & \cdots & x_{2 n} \\
\vdots & \vdots & \ddots & \vdots \\
x_{n 1} & x_{n 2} & \cdots & x_{n n}
\end{array}\right]
$$

$$
V=\left[\begin{array}{cccc}
v_{11} & v_{12} & \cdots & v_{1 n} \\
v_{21} & v_{22} & \cdots & v_{2 n} \\
\vdots & \vdots & \ddots & \vdots \\
v_{n 1} & v_{n 2} & \cdots & v_{n n}
\end{array}\right]
$$

The elements in the matrix $X$ above have the same property as Eq.(10). Accordingly, the elements of the matrix $X$ must satisfy the following conditions:

$$
\begin{gathered}
x_{i j} \in[0,1], \quad i=1,2, \cdots, n, \quad j=1,2, \cdots, n \\
\sum_{i=1}^{n} x_{i j}=1, \quad j=1,2, \cdots, n \\
\sum_{j=1}^{n} x_{i j}=1, \quad i=1,2, \cdots, n .
\end{gathered}
$$

Since the position and velocity in the new fuzzy particle swarm model have been transformed to the form of matrices, they are updated by the new Eqs.(17) and (18) by applying matrix operations.

$$
\begin{gathered}
V(t+1)=w \otimes V(t) \oplus\left(c_{1} r_{1}\right) \otimes\left(X^{\#}(t) \ominus X(t)\right) \\
\oplus\left(c_{2} r_{2}\right) \otimes\left(X^{*}(t) \ominus X(t)\right) \\
X(t+1)=X(t) \oplus V(t+1)
\end{gathered}
$$

The position matrix may violate the constraints (14), (15) and (16) after some iterations, thus it is necessary to normalize the position matrix. First we make all the negative elements in the matrix to become zero. If all elements in a column of the matrix are zero, they need be re-evaluated using a series of random numbers within the interval $[0,1]$. Then the matrix undergoes normalization, which makes all the rows and the columns sum up to 1 .

Since the position matrix indicates the potential assignment solution, we can "defuzzify" it to the feasible solution. We choose the element, which has the maximum value in the column, then tag it as " 1 ", and other numbers in the column and row are set as " 0 " in the assigning matrix. After all the columns and rows have been processed, we get the assignment solution without violating the constraints (14), (15) and (16), and then calculate the assignment cost of the solution.

In order to apply the PSO strategy for solving multiobjective quadratic assignment problems, the original scheme has to be modified. The algorithm need to search a set of different solutions (the so-called Pareto optimal set) instead of a single solution (as in global optimization) [13]. Based on the fuzzy particle swarm mapping, we apply multiobjective particle swarm optimization algorithm [12] to search toward the true Pareto front (non-dominated solutions) or approximate the Pareto optimal set for the mQAPs. Instead of the single objective particle swarm optimization, 
our algorithm has a solution pool to store the non-dominated solutions found by searching up to time $t$. Any of the solutions in the pool can be used as the global best particle to guide other particles in the swarm during the iterated process. The plot of the objective functions whose nondominated solutions are in the solution pool would make up of the Pareto front. The pseudo-code for the multi-objective particle swarm optimization algorithm is illustrated in Algorithm 1 .

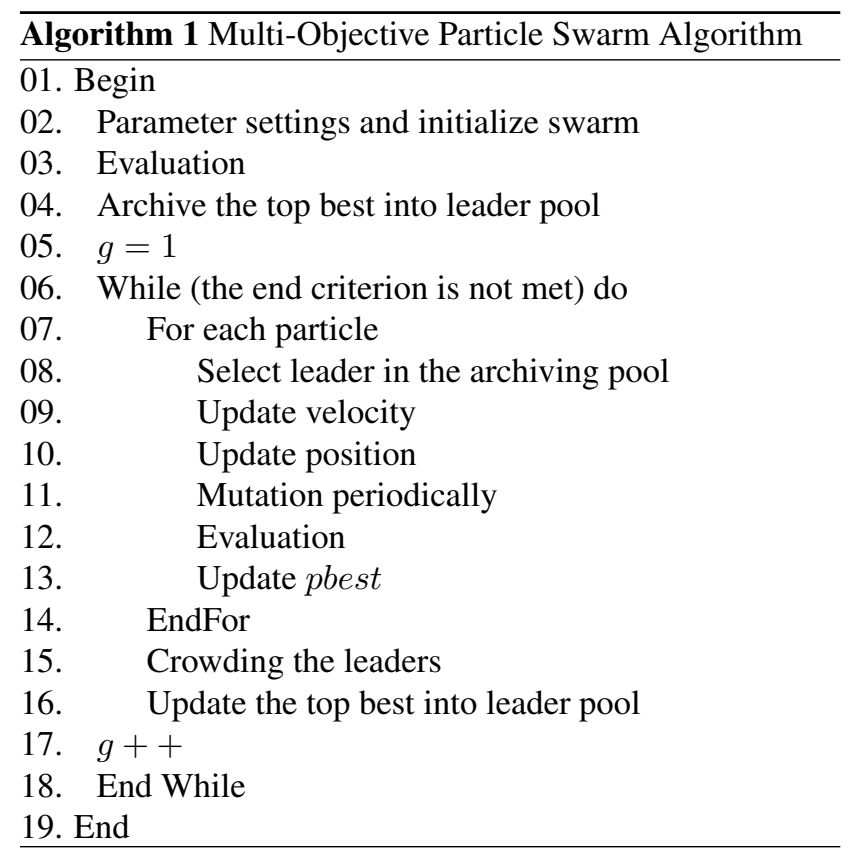

\section{Experiment and Results}

To illustrate the effectiveness and performance of the particle swarm optimization algorithm, we consider the instances from Knowles's datasets ${ }^{1}$. In our experiments, specific parameter settings for the algorithm are described in Table 1, where $D$ is the dimension of search space. The instances and associated Pareto fronts are provided in Table 2 and Figures 1 to 10 . It is noted that all the Pareto fronts are well-distributed, since our fuzzy scheme and matrix mapping provide higher probability of generating very diverse solutions for the problems during the defuzzification process from the particles' positions to the solutions.

\section{Conclusions}

In this paper, we presented a fuzzy particle swarm algorithm to handle the mQAP. In the fuzzy scheme, the representations of the position and velocity of the particles in
Table 1. Parameter settings for the algorithm.

\begin{tabular}{ll}
\hline Parameter name & Parameter value \\
\hline Swarm size & $\operatorname{int}(10+2 \operatorname{sqrt}(D))$ \\
Self coefficient $c_{1}$ & $0.5+\log (2)$ \\
Social coefficient $c_{2}$ & $0.5+\log (2)$ \\
Inertia weight $w$ & 0.91 \\
Maximum Iteration & $2 D$ \\
\hline
\end{tabular}

Table 2. Test Suite used.

\begin{tabular}{lllll}
\hline $\begin{array}{l}\text { Instance } \\
\text { name }\end{array}$ & $\begin{array}{l}\text { Instance } \\
\text { category }\end{array}$ & $\begin{array}{l}\text { Loc. } \\
\text { no. }\end{array}$ & $\begin{array}{l}\text { Flow mat. } \\
\text { no. }\end{array}$ & $\begin{array}{l}\text { Pareto } \\
\text { front }\end{array}$ \\
\hline KC10-2fl-1rl & Real-like & 10 & 2 & Figure 1 \\
KC10-2fl-3rl & Real-like & 10 & 2 & Figure 2 \\
KC10-2fl-5rl & Real-like & 10 & 2 & Figure 3 \\
KC20-2fl-2rl & Real-like & 20 & 2 & Figure 4 \\
KC20-2fl-3rl & Real-like & 20 & 2 & Figure 5 \\
KC20-2fl-3uni & Uniform & 20 & 2 & Figure 6 \\
KC20-2fl-4rl & Real-like & 20 & 2 & Figure 7 \\
KC20-2fl-5rl & Real-like & 20 & 2 & Figure 8 \\
KC30-3fl-1rl & Real-like & 30 & 3 & Figure 9 \\
KC30-3fl-2rl & Real-like & 30 & 3 & Figure 10 \\
\hline
\end{tabular}

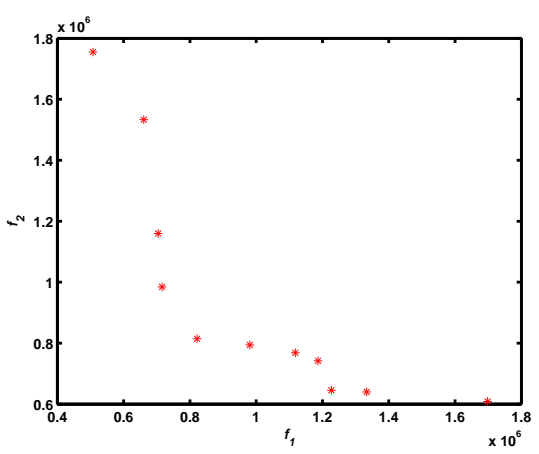

Figure 1. Pareto front for KC10-2fl-1rl.

\footnotetext{
${ }^{1}$ http://dbkgroup.org/knowles/mQAP
} 


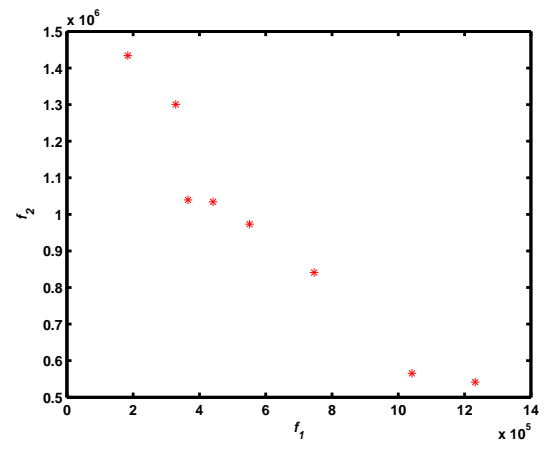

Figure 2. Pareto front for KC10-2fl-3rl.

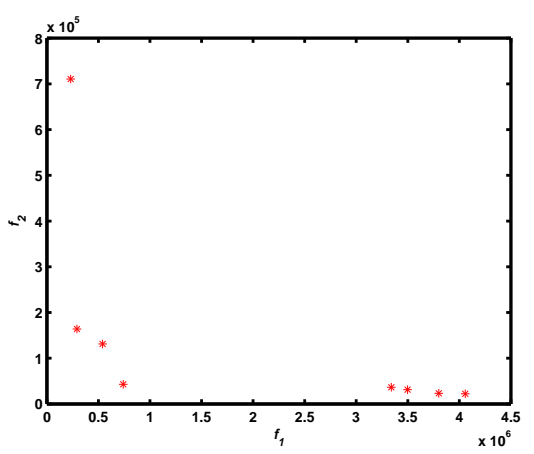

Figure 3. Pareto front for KC10-2fl-5rl.

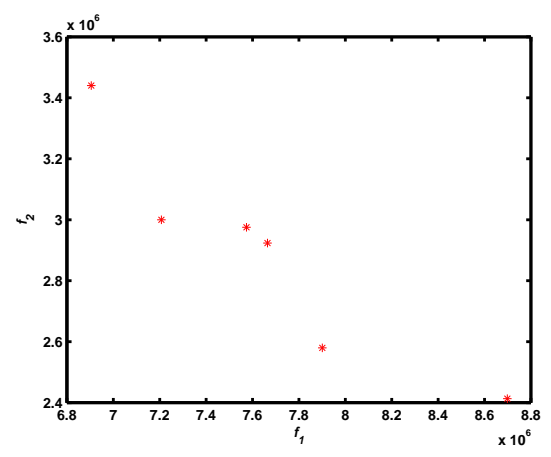

Figure 4. Pareto front for KC20-2fl-2rl.

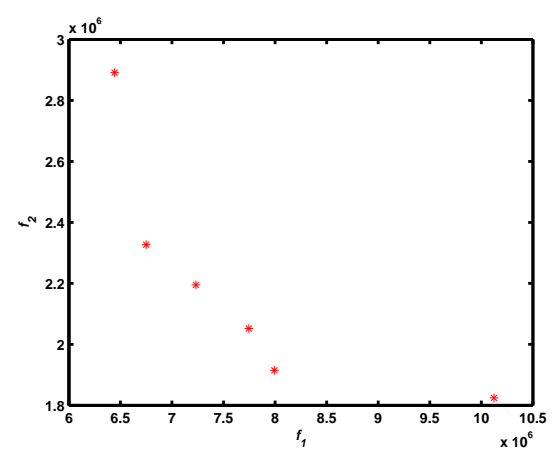

Figure 5. Pareto front for KC20-2fl-3rl.

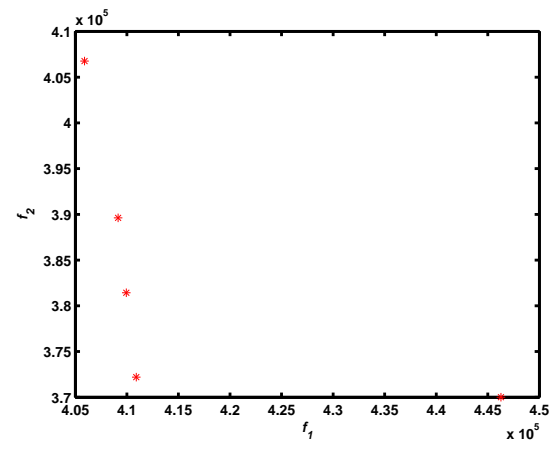

Figure 6. Pareto front for KC20-2fl-3uni.

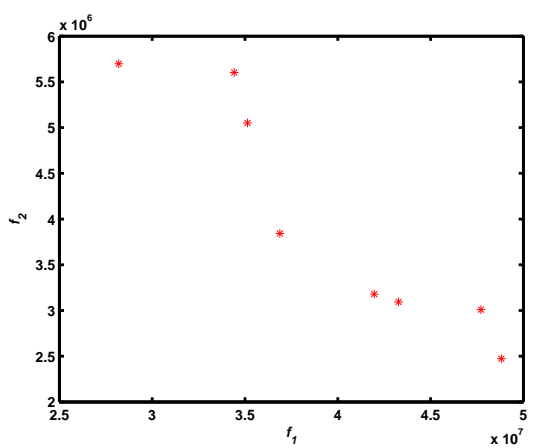

Figure 7. Pareto front for KC20-2fl-4rl.

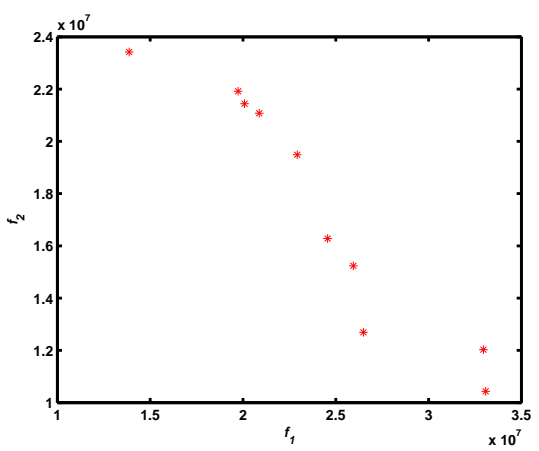

Figure 8. Pareto front for KC20-2fl-5rl.

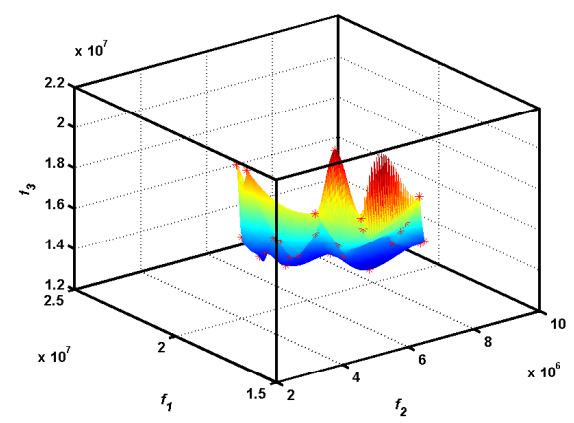

Figure 9. Pareto front for KC30-3fl-1rl. 


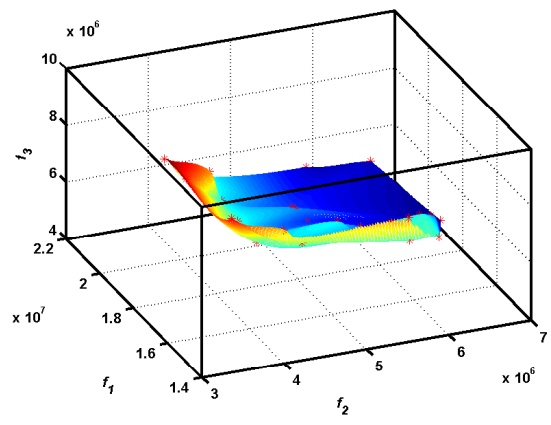

Figure 10. Pareto front for KC30-3fl-2rl.

the conventional PSO is extended from the real vectors to fuzzy matrices. We illustrated our approach by solving four benchmark mQAP instances and their Pareto fronts were provided. The Pareto fronts are well-distributed. Empirical results illustrate that the approach can be applied for solving mQAPs very effectively.

Our future work is targeted to generate more mQAP instances and involve more heuristics including hybrid approaches [14], [15].

\section{Acknowledgments}

This work is supported partly by NSFC (60573087) and MOST (2005CB321904).

\section{References}

[1] T. Koopmans and M. Beckman. Assignment Problems And The Location of Economic Activities. IEEE Econometrica, 25, pp. 53-76, 1957.

[2] J. Knowles and D. Corne. Towards Landscape Analyses To Inform The Design of A Hybrid Local Search for The Multiobjective Quadratic Assignment Problem. Soft Computing Systems: Design, Management and Applications, pp. 271-279. IOS Press, Amsterdam, 2002.

[3] R. Day and G. Lamont. Multiobjective Quadratic Assignment Problem Solved by an Explicit Building Block Search Algorithm - MOMGA-IIa. Lecture Notes in Computer Science (EvoCOP 2005), 3448, pp. 91-100, 2005.

[4] E. Loiola, N.M. Maia de Abreu, P.O. BoaventuraNetto, and T. Querido. A Survey For The Quadratic Assignment Problem. European Journal of Operational Research, 176, pp. 657-690, 2007.
[5] J. Knowles and D. Corne. Particle Swarm Optimization Versus Genetic Algorithms For phased Array Synthesis. In Proceedings of the Second International Conference Evolutionary Multi-Criterion Optimization, pp. 295-310, 2003.

[6] J. Kennedy and R. Eberhart. Swarm Intelligence, Morgan Kaufmann, CA, 2001.

[7] C. Grosan and A. Abraham, Stigmergic Optimization: Inspiration, Technologies and Perspectives, Studies in Computational Intelligence, Springer Verlag, Germany, pp. 1-24, 2006.

[8] H. Liu, A. Abraham, and M. Clerc. Chaotic Dynamic Characteristics in Swarm Intelligence. Applied Soft Computing Journal, 7, pp. 1019-1026, 2007.

[9] A. Abraham, H. Guo, and H. Liu. Swarm Intelligence: Foundations, Perspectives and Applications. In Studies in Computational Intelligence, N. Nedjah and L. Mourelle (Eds.), pp. 3-25. Springer, 2006.

[10] T. Stützle. Iterated Local Search for the Quadratic Assignment Problem. European Journal of Operational Research, 174, pp. 1519-1539, 2006.

[11] W. Pang, K. Wang, C. Zhou, and L. Dong. Fuzzy Discrete Particle Swarm Optimization For Solving Traveling Salesman Problem. In Proceedings of the Fourth International Conference on Computer and Information Technology, pp. 796-800, IEEE CS Press, 2004.

[12] C. Coello, G. Pulido, and M. Salazar. Handling Multiobjectives With Particle Swarm Optimization. IEEE Transactions on Evolutionary Computation, 8, pp. 256-279, 2004.

[13] A. Abraham, L. Jain and R. Goldberg (Eds.), Evolutionary Multiobjective Optimization: Theoretical Advances and Applications, Springer Verlag, London, ISBN 1852337877, 12 Chapters, 2005.

[14] C. Grosan, A. Abraham and M. Nicoara, Search Optimization Using Hybrid Particle Sub-Swarms and Evolutionary Algorithms, International Journal of Simulation Systems, Science and Technology, UK, Volume 6, Nos. 10 and 11, pp. 60-79, 2005.

[15] C. Grosan, Improving the performances of evolutionary algorithms for the multiobjective 0/1 knapsack problem using epsilon -dominance. Congress on Evolutionary Computation (CEC), G. Greenwood et al. (Eds.), IEEE Press, pp. 1958-1963, Portland, USA, 2004. 